\title{
The Effectiveness of Transdermal Iron Patches in Athletes With Suboptimal Iron Status (Part 1)
}

\author{
Rachel McCormick \\ The University of Western Australia \\ and The Western Australian \\ Institute of Sport \\ Leanne Lester \\ The University of Western Australia
}

\author{
Brian Dawson \\ The University of Western Australia \\ Carmel Goodman \\ The Western Australian \\ Institute of Sport
}

\author{
Marc Sim \\ Edith Cowan University and The \\ University of Western Australia
}

\author{
Peter Peeling \\ The University of Western Australia \\ and The Western Australian \\ Institute of Sport
}

\begin{abstract}
The authors compared the effectiveness of two modes of daily iron supplementation in athletes with suboptimal iron stores: oral iron (PILL) versus transdermal iron (PATCH). Endurance-trained runners (nine males and 20 females), with serum ferritin concentrations $<50 \mu \mathrm{g} / \mathrm{L}$, supplemented with oral iron or iron patches for 8 weeks, in a parallel group study design. Serum ferritin was measured at baseline and fortnightly intervals. Hemoglobin mass and maximal oxygen consumption $\left(\mathrm{V}_{2} \mathrm{O}_{2}\right.$ ax $)$ were measured preintervention and postintervention in PATCH. A linear mixed effects model was used to assess the effectiveness of each mode of supplementation on sFer. A repeated-measures analysis of variance was used to assess hemoglobin mass and $\dot{\mathrm{VO}}_{2}$ max outcomes in PATCH. There was a significant time effect $(p<.001)$, sex effect $(p=.013)$, and Time $\times$ Group interaction $(p=.009)$ for $\mathrm{sFer}$. At Week 6, PILL had significantly greater sFer compared with PATCH $(15.27 \mu \mathrm{g} / \mathrm{L}$ greater in PILL; $p=.019)$. Serum ferritin was $15.53 \mu \mathrm{g} / \mathrm{L}$ greater overall in males compared with females $(p=.013)$. There were no significant differences in hemoglobin mass $(p=.727)$ or $\mathrm{V}_{2} \max (p=.929)$ preintervention to postintervention in PATCH. Finally, there were six complaints of severe gastrointestinal side effects in PILL and none in PATCH. Therefore, this study concluded that PILL effectively increased sFer in athletes with suboptimal iron stores, whereas PATCH showed no beneficial effects.
\end{abstract}

Keywords: gastrointestinal side effects, oral iron therapy, supplementation mode

Dietary intake alone is often insufficient to fulfill the iron demands of an athlete. This issue has manifested in the ongoing high rates of iron deficiency (ID) among male ( $3 \%$ to $11 \%)$ and female ( 15\% to 35\%) athletes (Sim et al., 2019). Symptoms of ID include lethargy, fatigue, and, in more severe cases, reduced work capacity (Haas \& Brownlie, 2001), which may impede training and performance outcomes in athletes. Iron balance is a challenge for athletes because they encounter additional mechanisms of iron loss during exercise, including sweating, hematuria, gastrointestinal (GI) bleeding, and hemolysis (Peeling et al., 2008). Negative iron balance ensues when athletes consume a suboptimal amount of dietary iron to counteract these losses. In addition, these losses can be difficult to restore due to the low bioavailability of dietary iron (15-35\% for heme and 2-20\% for nonheme; Craig, 1994) and the inability to endogenously replenish taxed iron stores (Sim et al.,

McCormick, Dawson, Lester, and Peeling are with the School of Human Sciences (Exercise and Sport Science), The University of Western Australia, Crawley, Western Australia, Australia. McCormick, Goodman, and Peeling are with the Western Australian Institute of Sport, Mount Claremont, Western Australia, Australia. Sim is with the School of Medical and Health Sciences, Edith Cowan University, Joondalup, Western Australia, Australia; and Medical School, Royal Perth Hospital Unit, The University Western Australia, Perth, Western Australia, Australia. McCormick (rmccormick@wais.org.au) is corresponding author.
2019). Furthermore, recent research has identified a link between exercise-induced inflammation and an increase in the primary iron regulatory hormone, hepcidin. Hepcidin is reported to peak at $3 \mathrm{hr}$ and remain elevated for $6 \mathrm{hr}$ postexercise, and is linked to suppressed dietary iron absorption and recycling by duodenal enterocytes and macrophages, respectively (Nemeth et al., 2004; Peeling et al., 2009). Therefore, athletes will often need to consider supplemental sources of iron, beyond dietary sources, to achieve healthy iron status.

Typically, oral iron supplementation is the first approach to iron replacement therapy beyond a nutritional intervention, with ferrous sulfate the most commonly prescribed oral iron therapy (Cook, 2005, Tolkien et al., 2015). Characteristically, athletes' serum ferritin (sFer) will increase $40-80 \%$ following an 8- to 12 -week supplementation period, consisting of $\sim 100 \mathrm{mg}$ of elemental iron daily (Dawson et al., 2006; Garvican et al., 2014). However, adverse side effects associated with oral iron therapy, predominantly GI distress, are frequently reported (CanceloHidalgo et al., 2013; Coplin et al., 1991; Tolkien et al., 2015), often provoking nonadherence and ultimately treatment failures. Furthermore, there are concerns that soluble oral iron may be destructive to colonic microbiota, and that luminal iron may be a risk factor for inflammatory signaling (Werner et al., 2011). As such, contemporary research endeavors to ascertain strategies of iron 
supplementation that optimize iron absorption and reduce adverse side effects. Beyond oral iron therapy, iron delivery bypassing the gut is a promising prospect because it circumvents the side effects and absorption issues of the conventional therapy.

Parenteral iron therapy has been shown to be very effective at improving athletes' iron status, with $200-400 \%$ increases in sFer reported from 300 to $550 \mathrm{mg}$ of intravenous (IV) iron delivered across 6 weeks (Garvican et al., 2014). In the following, recent research pursues novel and alternate strategies of iron supplementation that bypass the gut, without the invasive procedure of IV administration, and has identified transdermal iron delivery as a potential strategy. The primary barriers of transdermal iron delivery include the reactive nature of free systemic iron and the low lipophilic permeability of the skin (Rejinold et al., 2019); however, ferric pyrophosphate was recently identified as an iron source suitable for transdermal administration (Gupta \& Crumbliss, 2000). Currently, a commercially available transdermal iron patch is being advertised as an alternative mode of iron supplementation, despite no existing data to support the efficacy of such an approach. Therefore, this investigation sought to be the first to compare the effectiveness of transdermal iron therapy via an iron patch, with oral iron supplementation, to elucidate the noninvasive treatment feasibility for ID athletes.

\section{Methods}

\section{Participants}

A total of 29 endurance-trained runners (nine males and 20 females) with suboptimal iron status (defined as sFer levels $<50 \mu \mathrm{g} / \mathrm{L}$; Peeling et al., 2014) were recruited for this study. Preparticipation conditions required participants not to be supplementing with iron within 3 weeks of commencing the study. Participants were informed of the purpose, requirements, and risks associated with their involvement. Written informed consent was obtained prior to study commencement. Ethics approval was obtained from the Human Research Ethics Committee of The University of Western Australia (RA/4/ 1/9030).

\section{Experimental Overview}

The experimental approach used in this study required athletes to supplement with iron for 8 weeks in a parallel group study design (Figure 1). Potential candidates undertook a rested preinvestigation blood measurement to confirm suboptimal iron status. Subsequently, eligible participants were assigned to one of two groups: (a) a treatment group that supplemented with a daily oral iron supplement (PILL) for 8 weeks or (b) a treatment group that supplemented with a transdermal iron supplement (PATCH) for 8 weeks. Participant groups were matched by sFer concentration.
The impact of the iron supplementation intervention on iron status was assessed via measurements of sFer concentration at the commencement, and at fortnightly intervals, throughout the 8-week training period (total of five blood samples). Participants acted as their own control via comparisons in sFer response with baseline.

During the supplementation period, the PILL group were required to consume their iron supplement upon waking to optimize iron absorption (McCormick et al., 2019), documenting the time of day of consumption in a daily supplement and training log. These participants were asked not to consume dairy-based food, tea, or coffee within 60 min of ingesting the iron supplement. The PATCH group was required to apply one patch overnight (for $8 \mathrm{hr}$ ) to bare skin superficial of the upper trapezius muscle, according to the manufacturer's directions (Iron Plus; PatchMD, Las Vegas, NV). All participants were encouraged to report any supplementation side effects they may have experienced in an open-ended comments box on each day of the supplement log and were also required to complete a 4-day food diary in the initial, mid, and final fortnight of the intervention period. In addition to the fortnightly sFer measures, a graded exercise test (GXT) and hemoglobin mass $\left(\mathrm{Hb}_{\text {mass }}\right)$ measurement were undertaken prior to and after the 8-week supplementation period in the PATCH group to further explore the effectiveness of the iron patches.

\section{Experimental Procedures}

Iron supplementation. During the intervention period, PILL participants supplemented with one Ferro Grad C tablet (Mylan Health Pty Ltd., Millers Point, NSW, Australia) upon waking daily (DAY). Each tablet contained $325 \mathrm{mg}$ of ferrous sulfate and $500 \mathrm{mg}$ of ascorbic acid, equating to a dose of $105 \mathrm{mg}$ of elemental iron. The PATCH group supplemented with one Iron Plus supplement patch (PatchMD) for $8 \mathrm{hr}$ every night of the intervention. Each patch contains $45 \mathrm{mg}$ of iron in the form of iron bisglycinate.

Blood collection. Venous blood was collected and analyzed for sFer by a commercial pathology laboratory (Clinipath Pathology, Osborne Park, WA, Australia) at baseline and fortnightly intervals during the intervention. Participants were instructed to visit the phlebotomy practice for a morning blood sample in a rested, nonfasting state (i.e., no morning exercise prior to blood collection). Participants still consumed their oral iron supplement on the morning blood was collected.

Hemoglobin mass. Hemoglobin mass was assessed using the optimized 2-min carbon monoxide rebreathing technique, as outlined by Schmidt and Prommer (2005). Determination of carboxyhemoglobin $(\% \mathrm{HbCO})$ was measured at baseline, plus $7 \mathrm{~min}$ after rebreathing, from capillary fingertip blood samples tested with an ABL80 blood gas analyzer (Radiometer, Copenhagen, Denmark).

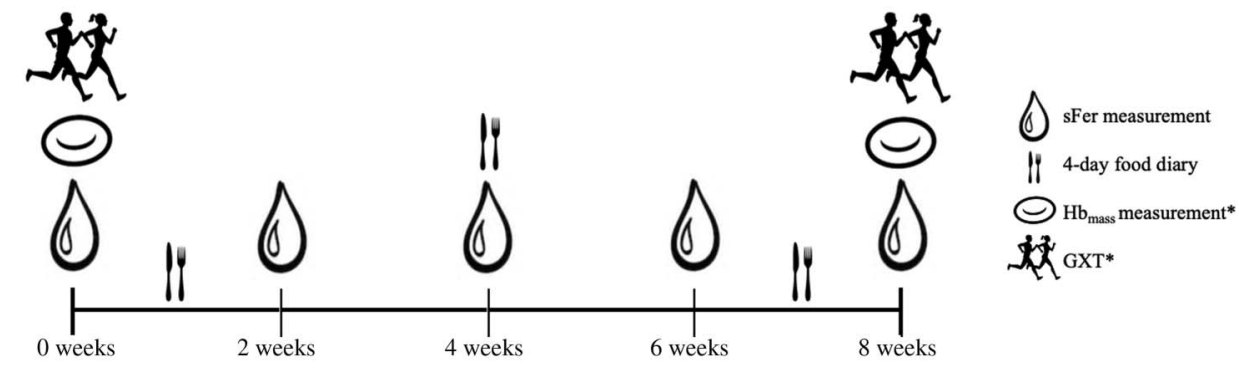

Figure 1 - Diagrammatic representation of experimental overview. Note. $\mathrm{sFer}=$ serum ferritin; $\mathrm{GXT}=$ graded exercise test; $\mathrm{Hb}$ mass $=$ hemoglobin mass. *PATCH group only. 
Hemoglobin mass was calculated from the mean change in $\%$ $\mathrm{HbCO}$ before and after carbon monoxide rebreathing. All $\mathrm{Hb}_{\text {mass }}$ measurements were conducted by the same technician.

Graded exercise test. The running GXT was conducted on a motorized treadmill (h/p/Cosmos Venus 200/100r; h/p/Cosmos Sports \& Medical Gmbh, Nußdorf, Germany), utilizing 3-min work and 1-min rest periods. The initial work velocity was set to $11.5 \pm 1.3 \mathrm{~km} / \mathrm{hr}$ with subsequent $1 \mathrm{~km} / \mathrm{hr}$ increments over each work period until volitional exhaustion. During the GXT, ventilation and expired air was analyzed for concentrations of $\mathrm{O}_{2}$ and $\mathrm{CO}_{2}$ using a TrueOne 2400 metabolic measurement system (Parvo Medics, Salt Lake City, UT). This system was calibrated pretest according to the manufacturer's specifications. The maximal oxygen consumption $\left(\dot{\mathrm{VO}}_{2} \max \right)$ was determined as highest $30 \mathrm{~s} \dot{\mathrm{VO}}_{2}$ reached during the final 3 min of the GXT.

Training load, dietary and menstrual monitoring. Participants were required to document their daily exercise, necessitating a measure of duration $(\mathrm{min})$, distance $(\mathrm{km})$, and a rating of perceived exertion (Borg, 1982) with Anchors 6 (no exertion) and 20 (maximal exertion). These data were used to calculate a daily training impulse (Foster et al., 2001) as a measure of training load. The training journal also included a section for female athletes to record their menstruation. All participants were also required to complete a 4-day food diary in the initial, mid, and final fortnight of the intervention period using the mobile diet tracking app, Easy Diet Diary (version 5.0.22; Xyris Software, Spring Hill, QLD, Australia). Participants attended an information session with a sports dietitian, prior to the commencement of the study, to familiarize themselves with portion measurements and the Easy Diet Diary app. This information was then analyzed using the nutritional analysis software, Foodworks (version 9.0.3871; Xyris Software; AusBrands 2017 and AusFoods 2017 databases).

\section{Statistical Analysis}

All diet and training data were initially analyzed using a two-way, repeated-measures analysis of variance to check for any differences between the PILL and PATCH groups. Linear mixed effects models were then used to assess the effectiveness of PILL and PATCH iron supplementation treatments on sFer. Serum ferritin was analyzed relative to time, treatment (PILL or PATCH), and sex using linear mixed effects models with a random intercept for each participant. Covariates considered were training load, energy intake, and (dietary) iron consumption. Initial models included all possible interactions, but nonsignificant interactions, except Time $\times$ Group, were dropped from the models for ease of interpretation. A repeated-measures analysis of variance was also used to assess the within-group differences in $\mathrm{Hb}_{\text {mass }}$ and $\mathrm{V}_{2}$ max measures over the intervention in the PATCH group. Results are expressed as mean and 95\% confidence intervals. The alpha level was defined as $\alpha \leq .05$.

\section{Results}

\section{Group Demographics}

Treatment group demographics are presented in Table 1. The PILL group consisted of 14 participants (six males and eight females) and consumed $55 \pm 3$ iron supplements (98.7\% compliance: 5,824 $\pm 348 \mathrm{mg}$ of elemental iron) throughout the 8-week intervention period. The PATCH group consisted of 14 participants (three males and 11 females) and applied 56 iron patches (99.5\% compliance:
Table 1 Group Characteristics for PILL and PATCH Treatments

\begin{tabular}{lccc}
\hline Treatment & $\begin{array}{c}\text { Age } \\
\text { (years) }\end{array}$ & $\begin{array}{c}\text { Body } \\
\text { mass (kg) }\end{array}$ & $\begin{array}{c}\text { Serum } \\
\text { ferritin } \\
(\boldsymbol{\mu g} / \mathbf{L})\end{array}$ \\
\hline PILL & & & \\
Female $(n=9)$ & $27 \pm 6$ & $61.7 \pm 7.4$ & $23 \pm 12$ \\
Male $(n=6)$ & $24 \pm 5$ & $69.3 \pm 2.5$ & $41 \pm 6$ \\
Overall & $26 \pm 6$ & $64.8 \pm 6.9$ & $31 \pm 13$ \\
PATCH & & & \\
Female $(n=11)$ & $27 \pm 5$ & $57.7 \pm 6.0$ & $33 \pm 10$ \\
Male $(n=3)$ & $26 \pm 5$ & $65.8 \pm 4.0$ & $37 \pm 14$ \\
Overall & $27 \pm 5$ & $59.5 \pm 6.5^{*}$ & $34 \pm 11$ \\
\hline
\end{tabular}

Note. Data are presented as mean $\pm S D$. PILL $=$ oral iron supplement; $\mathrm{PATCH}=$ transdermal iron supplement.

*A significant difference between groups $(p>.05)$.

\section{Table 2 Average Fortnightly Training Load and Average Dietary Intake for the PILL and PATCH Treatment}

\begin{tabular}{lcc}
\hline Variable & PILL & PATCH \\
\hline Fortnightly training load (AU) & $8,537 \pm 4,292$ & $7,915 \pm 3,821$ \\
Energy intake (kJ/day) & $10,765 \pm 3,254$ & $9,396 \pm 2,573$ \\
Carbohydrate intake (g/day) & $279 \pm 83$ & $248 \pm 79$ \\
Protein intake (g/day) & $107 \pm 28$ & $98 \pm 27$ \\
Fat intake (g/day) & $104 \pm 41$ & $85 \pm 23$ \\
Alcohol intake (g/day) & $3.59 \pm 7.69$ & $4.86 \pm 5.93$ \\
Dietary iron intake (mg/day) & $15 \pm 9$ & $12 \pm 5$ \\
\hline
\end{tabular}

Note. Data are presented as mean $\pm S D$. PILL $=$ oral iron supplement; $\mathrm{PATCH}=$ transdermal iron supplement.

$2,507 \pm 21 \mathrm{mg}$ of elemental iron) throughout the 8-week intervention period. There were no significant differences in age or baseline sFer between groups; however, there was a significant difference in body mass between groups $(p=.043)$. Of note, two female athletes did not report menstruating during the 8 -week intervention, though one of these instances was a secondary effect of contraception.

\section{Training and Dietary Analysis}

Table 2 presents the training load and nutrient intake of the PILL and PATCH treatment groups during the 8-week intervention. There was no time, group, or Time $\times$ Group effect for fortnightly training load, energy, protein, fat, $\mathrm{CHO}$, or dietary iron intake (all $\mathrm{ps}>.05$ ).

\section{Serum Ferritin}

Fortnightly sFer concentrations are depicted in Figure 2, alongside a more comprehensive depiction of individuals' preintervention and postintervention outcomes in Figure 3. There was a significant time effect $(p<.001)$, sex effect $(p=.013)$, and Time $\times$ Group interaction $(p=.009)$ for sFer. Our model indicates that by 6 weeks, the PILL group had a significantly greater sFer compared with the PATCH group $(15.27 \mu \mathrm{g} / \mathrm{L}$ greater in PILL; $p=.019 ; 95 \%$ confidence interval: $2.83<\mu<19.78 \mu \mathrm{g} / \mathrm{L})$. Serum ferritin was also 


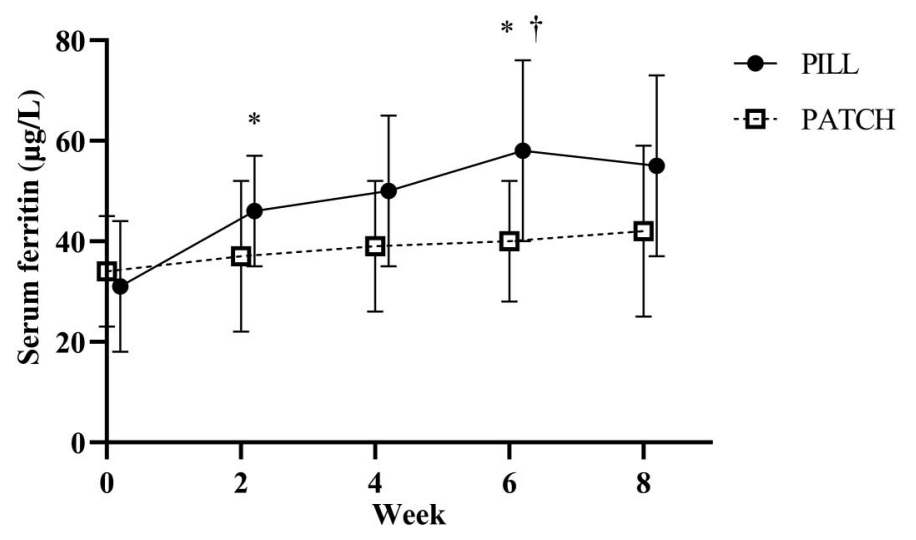

Figure 2 - Fortnightly serum ferritin concentrations for the oral (PILL) and transdermal $(\mathrm{PATCH})$ iron supplement treatment groups. Data are presented as mean $\pm S D$. *A significant increase from the preceding fortnight $(p<.05) .{ }^{\dagger}$ A significant difference between groups $(p<.05)$.

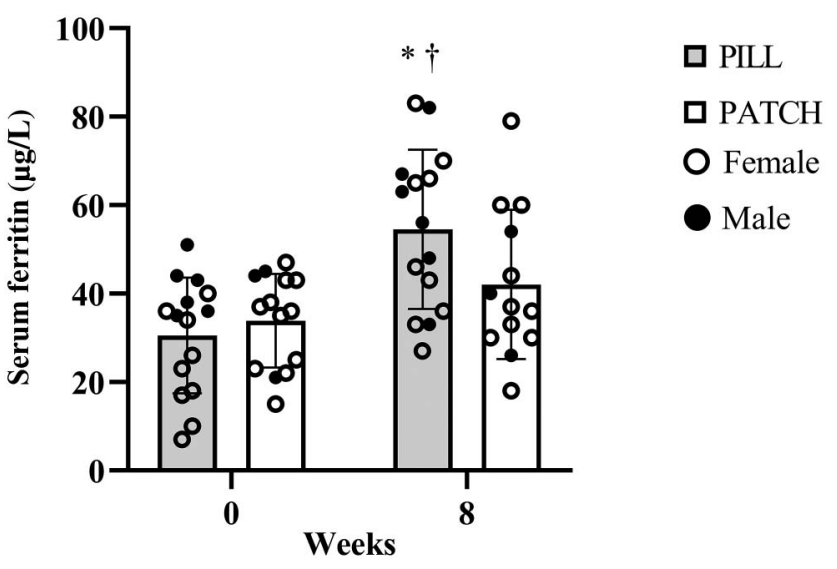

Figure 3 - Preintervention and postintervention serum ferritin concentrations for males and females in the oral (PILL) and transdermal (PATCH) iron supplement treatment groups. Group data are presented as mean $\pm S D$. $*$ A significant increase from baseline $(p<.05)$. ${ }^{\dagger}$ A significant difference between groups $(p<.05)$.

$15.53 \mu \mathrm{g} / \mathrm{L}$ greater overall in males compared with females ( $p=.013 ; 95 \%$ confidence interval: $3.55<\mu<27.52 \mu \mathrm{g} / \mathrm{L})$. No other covariates appeared to effect sFer.

\section{Hemoglobin Mass}

There was no significant difference in $\mathrm{Hb}_{\text {mass }}$ preintervention to postintervention $(652 \pm 125$ and $650 \pm 134 \mathrm{~g}$, respectively) in the PATCH group $(p=.727)$.

\section{Maximal Oxygen Consumption}

There was no significant difference in $\mathrm{V}_{2}$ max preintervention to postintervention $(56.06 \pm 9.09$ and $55.97 \pm 9.73 \mathrm{ml} / \mathrm{min} / \mathrm{kg}$, respectively) in the PATCH group $(p=.929)$.

\section{Adverse Side Effects Outcomes}

One participant from the PILL group withdrew from the study due to debilitating GI side effects. There were six further complaints of severe GI side effects in the PILL group, encompassing nausea $(n=1)$, stomach cramps $(n=2)$, and constipation $(n=3)$. There were no reported adverse effects in the PATCH group.

\section{Discussion}

Parenteral iron delivery techniques, such as IV administration, effectively increase athletes' sFer (Garvican et al., 2014) and bypass the gut, circumventing the GI side effects associated with oral iron supplementation that often limit compliance. However, IV iron delivery is highly invasive and is typically reserved for more severe cases of ID in athletes (Sim et al., 2019). Therefore, the alternative strategy of transdermal delivery of iron is appealing and applicable to the athletic population because of its purported capacity to bypass the gut and because it is noninvasive. Iron patches are currently being advertised as an alternate mode of iron supplementation despite very little data existing on their efficacy. To our knowledge, this is the first study to investigate the effects of iron patches and the potential for transdermal iron supplementation in athletes with suboptimal iron stores. Nevertheless, the results of our study suggest that, unlike daily oral iron supplementation, daily use of a commercial iron patch for 8 weeks (as per manufacturer's recommendation) does not increase an athlete's iron stores.

Beyond diet alone, oral iron supplementation is typically the first approach to iron replacement therapy because it is relatively cheap, safe, and effective (Santiago, 2012). The 60\% increase in sFer observed in the PILL group of this study attests to the $40-80 \%$ increase in sFer that is characteristic of ID athlete cohorts following 8-12 weeks of daily iron supplementation $(\sim 100 \mathrm{mg}$ of elemental iron per day; Dawson et al., 2006; Garvican et al., 2014. Ferrous sulfate is currently the most commonly prescribed oral iron therapeutic; however, ferrous sulfate supplementation is renowned for its associated GI side effects that can lead to noncompliance and treatment failure (Tolkien et al., 2015). Correspondingly, there were six reports of ongoing GI distress in the PILL group. Side effects included nausea, stomach cramps, and constipation, with one participant even unable to endure the full term of supplementation. This highlights the incentive for developing alternate modes of iron supplementation. In contrast, despite showing no beneficiary effects on $\mathrm{sFer}, \mathrm{Hb}_{\text {mass }}$, or $\dot{\mathrm{VO}}_{2}$ max in our athlete population, there were no complaints of any adverse side effects in the PATCH group. Although it is not yet clear that this method of iron delivery is effective, the absence of adverse side effects warrants further research into transdermal iron supplements.

Contemporary research is endeavoring to devise a viable transdermal iron therapy due to the high rates of noncompliance and GI toxicity associated with oral iron therapy (Gupta \& Crumbliss, 2000). Ferric pyrophosphate was recently identified as an iron source suitable for transdermal administration (Gupta \& Crumbliss, 2000) because it does not liberate free iron, is able to directly transfer iron to transferrin, and is capable of triggering iron transfer between transferrin molecules and between transferrin and ferritin (Morgan, 1977; Konopka et al., 1980). Unfortunately, its high molecular weight $(745 \mathrm{Da})$ and hydrophilicity resulted in poor passive permeation across the skin (Murthy \& Vaka, 2009). Nevertheless, the transdermal delivery of ferric pyrophosphate was found to be enhanced by the electrically mediated technique, iontophoresis (Murthy \& Vaka, 2009), and microporation via a soluble microneedle system (Modepalli et al., 2016). Of note, the commercially available iron patch used in the present study is a small topical patch containing iron bisglycinate (molecular weight: $204 \mathrm{Da}$ ) that is alleged to absorb passively over $8 \mathrm{hr}$; however, our 
$\mathrm{sFer}$ and $\mathrm{Hb}_{\text {mass }}$ data did not support this supposition. Iron bisglycinate is an amino acid chelate that has been identified as an ideal food fortificant because of its high bioavailability and low reactivity (van Stuijvenberg et al., 2006), but like ferric pyrophosphate, it may require penetration enhancement to successfully be delivered transdermally. Our study outcomes suggest that the assumption of passive transdermal iron absorption cannot be relied upon and that future research needs to establish an effective transdermal iron transport mechanism before it can be marketed as an effective iron supplementation strategy.

The capability of the passive delivery mechanism to penetrate the skin barrier is likely the primary limiting factor to the outcomes of the PATCH group; however, there was also a clear discrepancy in the dosage of iron between the PILL and PATCH groups (105 and $45 \mathrm{mg}$, respectively). Nevertheless, although the PILL group ingested $105 \mathrm{mg}$ of elemental iron daily, the bioavailability of iron is low, and Moretti et al. (2015) previously demonstrated that fractional absorption decreases as oral iron dosage increases. In fact, Moretti et al. (2015) revealed that $\sim 19 \%$ of an $80 \mathrm{mg}$ oral dose of iron is functionally absorbed, which, in the context of our study, would equate to, at best, $\sim 20 \mathrm{mg}$ of iron being functionally absorbed daily in the PILL group. In comparison, the PATCH group received $45 \mathrm{mg}$ delivered transdermally, and while the bioavailability of this mechanism remains unknown, our data would suggest that it is very low and ineffective for athletes with suboptimal iron.

Although our work appears to be the first study to investigate the effectiveness of iron patches in athletes, Saurabh et al. (2019) recently assessed the multivitamin patch in gastric bypass patients. Here, these authors found that patients using the multivitamin patch had twice the frequency of vitamin deficiencies after 12 months, and concluded that the transdermal multivitamins were not as effective as oral vitamin supplements. In parallel, the data from the present study indicate that it is currently advantageous for athletes with suboptimal iron status to supplement with oral iron. However, a recent review describing the latest advances and novel approaches to transdermal vitamin delivery techniques highlights promising theoretical formulations but a lack of sufficient clinical and preclinical assessments (Rejinold et al., 2019). Therefore, future research should endeavor to improve therapeutic delivery or iron, and other vitamins, because the noninvasive procedure and potential for reductions to GI sensitivity may be applicable to several populations.

Finally, this investigation highlights the relatively greater prevalence and severity of ID in female, compared with male, athletes (Figure 3). Males represented $31 \%$ of participants, and there was a clear distinction in baseline sFer between females $(29 \pm 12 \mu \mathrm{g} / \mathrm{L})$ and males $(39 \pm 8 \mu \mathrm{g} / \mathrm{L})$. However, this may be elucidated, in part, by the dietary iron intakes captured in the present study, with only $10 \%$ of female athletes consuming the recommended dietary iron intake of $\geq 18 \mathrm{mg} /$ day, whereas all male athletes consumed $\geq 8 \mathrm{mg} /$ day. This would suggest that females may be less likely than males to achieve a daily iron intake (and/or energy intake) adequate to sustain both training and reproductive demands, increasing their vulnerability to ID.

In summary, this study demonstrates that daily oral iron supplementation for 8 weeks effectively increases sFer in athletes with suboptimal iron stores, although this treatment is accompanied with the well-documented GI side effects. In contrast, the transdermal iron patch showed no beneficiary effects on $\mathrm{sFer}, \mathrm{Hb}_{\text {mass }}$, or $\mathrm{VO}_{2}$ max in athletes with suboptimal iron stores. Consequently, we would advise athletes with compromised iron stores, who have initially addressed any dietary iron intake deficits, to continue to supplement with the conventional oral iron therapy, or in severe cases, to consider parenteral iron approaches under the direction of their physicians. In consideration of our companion study (Part 2), athletes with suboptimal iron may consider alternate day oral iron supplementation, as this was shown to increase sFer comparably with daily oral iron, and was associated with less GI side effects. Nevertheless, future research should continue to pursue the potential for effective transdermal iron supplementation to eliminate the limiting GI side effects.

\section{Acknowledgments}

This study was designed by R. McCormick, C. Goodman, and P. Peeling; the data were collected and analyzed by R. McCormick and L. Lester; data interpretation and manuscript preparation were undertaken by R. McCormick, B. Dawson, M. Sim, and P. Peeling. All authors approved the final version of the article. The authors thank all the participants involved in this study for their commitment to the iron supplement protocol. The authors declare no conflict of interest. The results of the study are presented clearly, honestly, and without fabrication, falsification, or inappropriate data manipulation.

\section{References}

Borg, G.A. (1982). Psychophysical bases of perceived exertion. Medicine \& Science in Sports \& Exercise, 14(5), 377-381. PubMed ID: 7154893

Cancelo-Hidalgo, M.J., Castelo-Branco, C., Palacios, S., Haya-Palazuelos, J., Ciria-Recasens, M., Manasanch, J., \& Pérez-Edo, L. (2013). Tolerability of different oral iron supplements: A systematic review. Current Medical Research Opinion, 29(4), 291-303. PubMed ID: 23252877 doi:10.1185/03007995.2012.761599

Cook, J.D. (2005). Diagnosis and management of iron-deficiency anaemia. Best Practice \& Research: Clinical Haematology, 18(2), 319-332.

Coplin, M., Schuette, S., Leichtmann, G., \& Lashner, B. (1991). Tolerability of iron: A comparison of bis-glycino iron II and ferrous sulfate. Clinical Therapeutics, 13(5), 606-612. PubMed ID: 1799918

Craig, W.J. (1994). Iron status of vegetarians. The American Journal of Clinical Nutrition, 59(5), 1233S-1237S. doi:10.1093/ajen/59.5.1233S

Dawson, B., Goodman, C., Blee, T., Claydon, G., Peeling, P., Beilby, J., \& Prins, A. (2006). Iron supplementation: Oral tablets versus intramuscular injections. International Journal of Sport Nutrition and Exercise Metabolism, 16(2), 180-186. PubMed ID: 16779924 doi:10. 1123/ijsnem.16.2.180

Foster, C., Florhaug, J.A., Franklin, J., Gottschall, L., Hrovatin, L.A., Parker, S., ... Dodge, C. (2001). A new approach to monitoring exercise training. Journal of Strength and Conditioning Research, 15(1), 109-115. PubMed ID: 11708692

Garvican, L.A., Saunders, P.U., Cardoso, T., Macdougall, I.C., Lobigs, L.M., Fazakerley, R., ... Gore, C.J. (2014). Intravenous iron supplementation in distance runners with low or suboptimal ferritin. Medicine \& Science in Sports \& Exercise, 46(2), 376-385. PubMed ID: 23872938 doi:10.1249/MSS.0b013e3182a53594

Gupta, A., \& Crumbliss, A.L. (2000). Treatment of iron deficiency anemia: Are monomeric iron compounds suitable for parenteral administration? Journal of Laboratory and Clinical Medicine, 136(5), 371-378. PubMed ID: 11079464 doi:10.1067/mlc.2000.110368

Haas, J.D., \& Brownlie, T. (2001). Iron deficiency and reduced work capacity: A critical review of the research to determine a causal relationship. Journal of Nutrition, 131(2), 676S-690S. doi:10.1093/ jn/131.2.676S 
Konopka, K., Mareschal, J.C., \& Crichton, R.R. (1980). Iron transfer from transferrin to ferritin mediated by pyrophosphate. Biochemical and Biophysical Research Communications, 96(3), 1408-1413. PubMed ID: 6254532 doi:10.1016/0006-291X(80)90107-2

McCormick, R., Moretti, D., McKay, A.K., Laarakkers, C.M., Vanswelm, R., Trinder, D., .. P Peeling, P. (2019). The impact of morning versus afternoon exercise on iron absorption in athletes. Medicine and Science in Sports and Exercise, 51(10), 2147-2155.

Modepalli, N., Shivakumar, H.N., McCrudden, M.T., Donnelly, R.F., Banga, A., \& Murthy, S.N. (2016). Transdermal delivery of iron using soluble microneedles: Dermal kinetics and safety. Journal of Pharmaceutical Sciences, 105(3), 1196-1200. PubMed ID: 26928401 doi:10.1016/j.xphs.2015.12.008

Moretti, D., Goede, J.S., Zeder, C., Jiskra, M., Chatzinakou, V., Tjalsma, H., ... Zimmermann, M.B. (2015). Oral iron supplements increase hepcidin and decrease iron absorption from daily or twicedaily doses in iron-depleted young women. Blood, 126(17), 19811989. PubMed ID: 26289639 doi:10.1182/blood-2015-05-642223

Morgan, E. (1977). Iron exchange between transferrin molecules mediated by phosphate compounds and other cell metabolites. Biochimica et Biophysica Acta, 499(1), 169-177. PubMed ID: 19087 doi:10.1016/ 0304-4165(77)90239-2

Murthy, S.N., \& Vaka, S.R. (2009). Irontophoresis: Transdermal delivery of iron by iontophoresis. Journal of Pharmaceutical Sciences, 98(8), 2670-2676. PubMed ID: 19117049 doi:10.1002/jps.21641

Nemeth, E., Rivera, S., Gabayan, V., Keller, C., Taudorf, S., Pedersen, B.K., \& Ganz, T. (2004). IL-6 mediates hypoferremia of inflammation by inducing the synthesis of the iron regulatory hormone hepcidin. Journal of Clinical Investigation, 113(9), 1271-1276. PubMed ID: 15124018 doi:10.1172/JCI200420945

Peeling, P., Dawson, B., Goodman, C., Landers, G., \& Trinder, D. (2008). Athletic induced iron deficiency: New insights into the role of inflammation, cytokines and hormones. European Journal of Applied Physiology, 103(4), 381-391. PubMed ID: 18365240 doi:10.1007/ s00421-008-0726-6

Peeling, P., Dawson, B., Goodman, C., Landers, G., Wiegerinck, E., Swinkels, D., \& Trinder, D. (2009). Effects of exercise on hepcidin response and iron metabolism during recovery. International Journal of Sport Nutrition and Exercise Metabolism, 19(6), 583-597. PubMed ID: 20175428 doi:10.1123/ijsnem.19.6.583
Peeling, P., Sim, M., Badenhorst, C., Dawson, B., Govus, A.D., Abbiss, C.R., ... Trinder, D. (2014). Iron status and the acute post-exercise hepcidin response in athletes. PLoS One, 9(3), e93002. PubMed ID: 24667393 doi:10.1371/journal.pone.0093002

Rejinold, N.S., Kim, H.K., Isakovic, A.F., Gater, D.L., \& Kim, Y.C. (2019). Therapeutic vitamin delivery: Chemical and physical methods with future directions. Journal of Controlled Release, 298, 83-98. PubMed ID: 30707902 doi:10.1016/j.jconrel.2019.01.038

Santiago, P. (2012). Ferrous versus ferric oral iron formulations for the treatment of iron deficiency: A clinical overview. The Scientific World Journal, 2012(4), 846824. PubMed ID: 22654638 doi:10.1100/2012/ 846824

Saurabh, S., Gao, Y., Maduka, S., Smith, L., Lasley, R., \& Singh, N. (2019). Is transdermal multivitamin patch effective in gastric bypass patients? Obesity Surgery, 29(12), 3818-3823. PubMed ID: 31302845 doi:10.1007/s11695-019-04070-5

Schmidt, W., \& Prommer, N. (2005). The optimised CO-rebreathing method: A new tool to determine total haemoglobin mass routinely. European Journal of Applied Physiology, 95(5-6), 486-495. PubMed ID: 16222540 doi:10.1007/s00421-005-0050-3

Sim, M., Garvican-Lewis, L.A., Cox, G.R., Govus, A., McKay, A.K., Stellingwerff, T., \& Peeling, P. (2019). Iron considerations for the athlete: A narrative review. European Journal of Applied Physiology, 119(7), 1463-1478. PubMed ID: 31055680 doi:10.1007/s00421019-04157-y

Tolkien, Z., Stecher, L., Mander, A.P., Pereira, D.I.A., \& Powell, J.J. (2015). Ferrous sulfate supplementation causes significant gastrointestinal side-effects in adults: A systematic review and metaanalysis. PLoS One, 10(2), e0117383. PubMed ID: 25700159 doi: 10.1371/journal.pone. 0117383

van Stuijvenberg, M.E., Smuts, C.M., Wolmarans, P., Lombard, C.J., \& Dhansay, M.A. (2006). The efficacy of ferrous bisglycinate and electrolytic iron as fortificants in bread in iron-deficient school children. British Journal of Nutrition, 95(3), 532-538. doi:10.1079/ BJN20051669

Werner, T., Wagner, S.J., Martínez, I., Walter, J., Chang, J.S., Clavel, T., ... Haller, D. (2011). Depletion of luminal iron alters the gut microbiota and prevents Crohn's disease-like ileitis. Gut, 60(3), 325-333. PubMed ID: 21076126 doi:10.1136/gut.2010. 216929 